\title{
Gravitational Waves Produced by Ejection of Jet Superluminal Components, Precession and Gravito-Magnetic Distortion of Accretion Disks in Active Galactic Nuclei, Micro-Quasars, and T-Tauri Stars Dynamically Driven by Bardeen-Petterson Effect
}

\author{
Herman J. Mosquera Cuesta ${ }^{0,1,2,3 *}$, Luis A. Sanchez ${ }^{4}$, Daniel Alfonso Pardo ${ }^{4}$, Anderson Caproni ${ }^{5}$ \\ and Zulema Abraham ${ }^{6}$
}

${ }^{\circ}$ Departmento de Física Universidade Estadual Vale do Acaráu, Avenida da Universidade 850, Campus da Betânia, CEP 62.040-370, Sobral, Ceará, Brazil, HJMC is fellow of the Ceará State Foundation for the Development of Science and Technology (FUNCAP), Fortaleza, CE, Brazil

${ }^{1}$ Instituto de Cosmologia, Relatividade e Astrofísica (ICRA-BR), Centro Brasileiro de Pesquisas Físicas, Rua Dr. Xavier Sigaud 150, CEP 22290-180, Urca Rio de Janeiro, RJ, Brazil

${ }^{2}$ International Center for Relativistic Astrophysics Network (ICRANet), International Coordinating Center, Piazzalle della Repubblica 10, 065112, Pescara, Italy

${ }^{3}$ International Institute for Theoretical Physics and Mathematics Einstein-Galilei, via Bruno Buozzi 47, 59100 Prato, Italy

${ }^{4}$ Escuela de Física, Universidad Nacional de Colombia, Sede Medellín, A.A. 3840, Medellín, Colombia

${ }^{5}$ Núcleo de Astrofísica Teórica (NAT), Pós-graduação e Pesquisa, Universidade Cruzeiro do Sul Rua Galvão Bueno 868, Liberdade 01506-000, São Paulo, SP, Brasil

${ }^{6}$ Instituto de Astronomia, Geofísica e Ciências Atmosfêricas, Universidade de São PauloRua do Matão 1226, Cidade Universitária, CEP 05508-900, São Paulo, SP, Brazil

\begin{abstract}
Jet superluminal components are recurrently ejected from active galactic nuclei, micro-quasars, T-Tauri star, and several other astrophysical systems. The mechanism driving this powerful phenomenon is not properly settled down yet. In this article we suggest that ejection of ultrarelativistic components may be associated to the superposition of two actions: precession of the accretion disk induced by the Kerr black hole $(\mathrm{KBH})$ spin, and fragmentation of tilted disk; this last being an astrophysical phenomenon driven by the general relativistic Bardeen-Petterson (B-P) effect. As fragmentation of the accretion disk takes place at the B-P transition radius, a suspended accretion state can develop amid this boundary and the innermost stable orbit around the $\mathrm{KBH}$, which drives a turbulent flow along the inner accretion disk. The torus distortion caused by both hydrodynamic coupling of the inner face to the outer face, and Maxwell stresses from the magnetic field makes it to generate gravitational waves from its turbulent flow in the suspended accretion state. This magneto-centrifugal barrier also precludes incoming matter to penetrate the inner disk, creating "en passant" a sort of force-free region. The incoming material trapped in this sort of Lagrange internal point will forcibly precess becoming a source of continuous, frequency-modulated gravitational waves. Eventually, a condition of orbital resonance (beating) can activate between the precession pace and the frequency of the warps travelling along the torus due to its differential rotation. As this transition radius behaves like a sort of Lagrange internal point in a force-free magnetosphere, at resonance blobs can be expelled at ultrarelativistic velocities from the B-P radius in virtue of the power provided by either the vertical (to the disk) linear momentum carried by the surface acoustic wave on the torus warps, or the Aschenbach effect in a nearly maximal $\mathrm{KBH}$, or some other orbital resonance like the well known resonance 3:1. The launching of superluminal components of jets should produce powerful gravitational wave $(\mathrm{GW})$ bursts during its early acceleration phase, which can be catched on the fly by current GW observatories. Here we compute the characteristic amplitude and frequency of such signals and show that they are potentially detectable by the GW observatory LISA.
\end{abstract}

Keywords: Galaxies: active -- galaxies: nuclei -- galaxies: quasars, black hole physics, magnetic fields, stars: micro-quasars -stars: individual (T-Tauri).

\section{ASTROPHYSICAL MOTIVATION}

Radio interferometric observations of active galactic nuclei (AGN - radio galaxies, blazars, Seyfert I and II galaxies, etc.), micro-quasars and T-Tauri stars exhibit apparently stationary bright cores enshrouded by faint haloes, kiloparsec $(\mathrm{kpc})$ jets, and superluminal parsec $(\mathrm{pc})$ components that appear to travel following curved trajectories with spatially changing velocities. High spatial

*Address correspondence to this author at the Instituto de Cosmologia, Relatividade e Astrofsica (ICRA-BR), Centro Brasileiro de Pesquisas Fsicas Rua Dr. Xavier Sigaud 150, CEP 22290-180 Urca, Rio de Janeiro, RJ, Brazil; Fax: 005521 21417266; Tel: 005521 21417377; E-mail: herman @ icra.it 
resolution observations of AGN show that the superluminal ejected material is moving out from their very central regions: the core. The resultant structure is seen in the sky as pairs of curved jets that extend from several pc, kpc, and even megaparsecs $(\mathrm{Mpc})$ distance scales from the AGN core. Jet material seems to be constituted of a plasma composed of electron-positron pairs plus some baryons. The jet morphology is diverse, some of them presenting features that resembles spirals in the sky. These characteristics were interpreted by several authors as due to helical motion of the components along the jet. In an alternative view [1,2], it was proposed that such helicoidal appearance of the jet may be due to precession of the disk-jet structure. A few other proposals to cope with the observations are at disposal, but the actual mechanism driving this phenomenology is nonetheless not yet conclusively established.

The first systematic study on the Bardeen-Petterson (B-P) effect as the potential driving engine (mechanism) behind the observed dynamics of AGN was presented in Ref. [3]. (See also the list of papers [4-7], in which we extend these results to the analysis of some other specific AGN sources, and to confront the B-P scenario for AGN with alternative mechanisms to explain the helicoidal motion of jet components and the disk warping). Therein we have shown that for a large sample of AGN the observed disk and/or jet precession can be consistently explained as driven by the B$\mathrm{P}$ effect. As described below, a torus encircling a rotating (Kerr) supermassive black hole (SMBH), as the state-of-theart envisions AGN, may lead a rotating SMBH into a dramatic dynamical stage, known as suspended accretion state (SAS) [8], in which most of the SMBH spin energy can be released through gravitational radiation due to the spindisk magnetic field coupling. As SAS can develop only in a very compact black hole (BH)-Torus structure [8], we argue in a paper related to this [9] that in the case of AGN the Bardeen-Petterson effect is a necessary and sufficient mechanism to produce SAS. ${ }^{1}$ In that related paper [9] we compute the emission of gravitational waves (GW) generated by the AGN torus dynamics as driven by the B-P effect.

In a tilted accretion disk orbiting around a rotating $\mathrm{BH}$, the B-P effect creates a gap, or discontinuity, which is known as transition region or B-P radius. Matter arriving from the outer part of the disk can pile-up at this radius because of the magneto-centrifugal barrier creating a sort of Lagrange internal point. Consequently, the accumulated material can condensate in a blob or knot whereby becoming a source of continuous GW due to its orbital motion around the central $\mathrm{BH}$ at the B-P radius. It also radiates $\mathrm{GW}$ upon being launched into space at ultrarelativistic velocity along the instantaneous jet direction (which explains why jets appear to be helicoidal, as any component ejection ramdonly points out to different directions in space). In this explanatory scenario for the observed AGN dynamics, we discuss the characteristics of those $\mathrm{GW}$ signals.

\footnotetext{
${ }^{1}$ It worths to notice that the physical mechanism that could give origin to disk breaking and precession in AGN and related astrophysical systems was not properly identified in Ref. [10,12]
}

\section{BARDEEN-PETTERSON EFFECT IN AGN}

The frame dragging produced by a Kerr black hole, known as Lense-Thirring effect, leads a particle to precess (and nutate) if its orbital plane intersects the $\mathrm{BH}$ equatorial plane, to which the $\mathrm{BH}$ angular momentum, $\vec{J}_{\mathrm{BH}}$, is perpendicular. The amplitude of the precession angular velocity decreases as the third power of the distance, i.e., $\omega_{\mathrm{L}-\mathrm{T}} \propto r^{-3}$, and becomes negligible at large distances from the KBH. If a viscous accretion disk is inclined with respect to the equatorial plane of the Kerr black hole, the differential precession will produce warps in the disk. The intertwined action of the Lense-Thirring effect and the internal viscosity of the accretion disk drives the disk to break apart at a special distance from the KBH known as Bardeen-Petterson radius, whilst the coupling of the gravitational rotating field to the inner disk angular momentum enforces the spin axis of the inner accretion disk to align with the angular momentum axis of the Kerr black hole, in a time scale which depends on several parameters of the system. This is known as the Bardeen-Petterson effect [11] and affects mainly the innermost part of the disk, while its outer parts tend to remain in its original configuration due to the short range of the Lense-Thirring effect. The transition region between these two regimes is referred to as B-P radius, and its exact location depends on the Mach number of the accretion disk. (The attentive reader is addressed to [3] for further details and a list of references on this astrophysical effect).

\subsection{Kerr Space-Time Compactness}

The disk dynamics caused by the B-P effect thus relates the total torque $\vec{T}$ applied to the accretion disk to the disk precession frequency $\omega_{\text {prec }}$ or period $P_{\text {prec }}$, and its total angular momentum $\vec{L}_{d}$. The fundamental relation turns out to be (see Ref. [3] for the derivation of this formula, and further details)

$$
\frac{P_{\text {prec }}}{M_{\text {BH }}}=K_{\text {P }} \frac{\int_{\xi_{\text {ms }}}^{\xi_{\text {out }}} \Sigma_{\mathrm{d}}(\xi)[\Upsilon(\xi)]^{-1} \xi^{3} d \xi}{\int_{\xi_{\text {ms }}}^{\xi_{\text {out }}} \Sigma_{\mathrm{d}}(\xi) \Psi(\xi)[\Upsilon(\xi)]^{-2} \xi^{3} d \xi}
$$

where $K_{\mathrm{P}}=1.5 \times 10^{-38} \mathrm{~s} \mathrm{~g}^{-1}, \Sigma_{\mathrm{d}}$ the disk surface density, $\xi=r / R_{\mathrm{g}} \quad$ a dimensionless variable, $\quad \xi_{\text {out }}=R_{\text {out }} / R_{\mathrm{g}}$, $\Upsilon(\xi)=\xi^{3 / 2}+a_{*}$ and $\Psi(\xi)=1-\sqrt{1-4 a_{*} \xi^{-3 / 2}+3 a_{*}^{2} \xi^{-2}}$, with $a_{*}$ the spin parameter of KBH .

As such, Eq. (1) is parameterized by the ratio of the precession period to the black hole mass, which in fact is related to a fundamental invariant of the Kerr metric (see discussion below). As one can see from Eq. (1), the precession period depends on how the disk surface density $\Sigma_{\mathrm{d}}$ varies with the radius. In what follows we take as an AGN model the source quasar 3C 345. In the search for a disk model driven by the B-P effect able to reproduce both the observed period and the inferred mass of the $\mathrm{BH}$ in $3 \mathrm{C}$ 345 , we have modelled the torus by using a power-law function such as $\Sigma_{\mathrm{d}}(\xi)=\Sigma_{0} \xi^{s}$, and also an exponential 

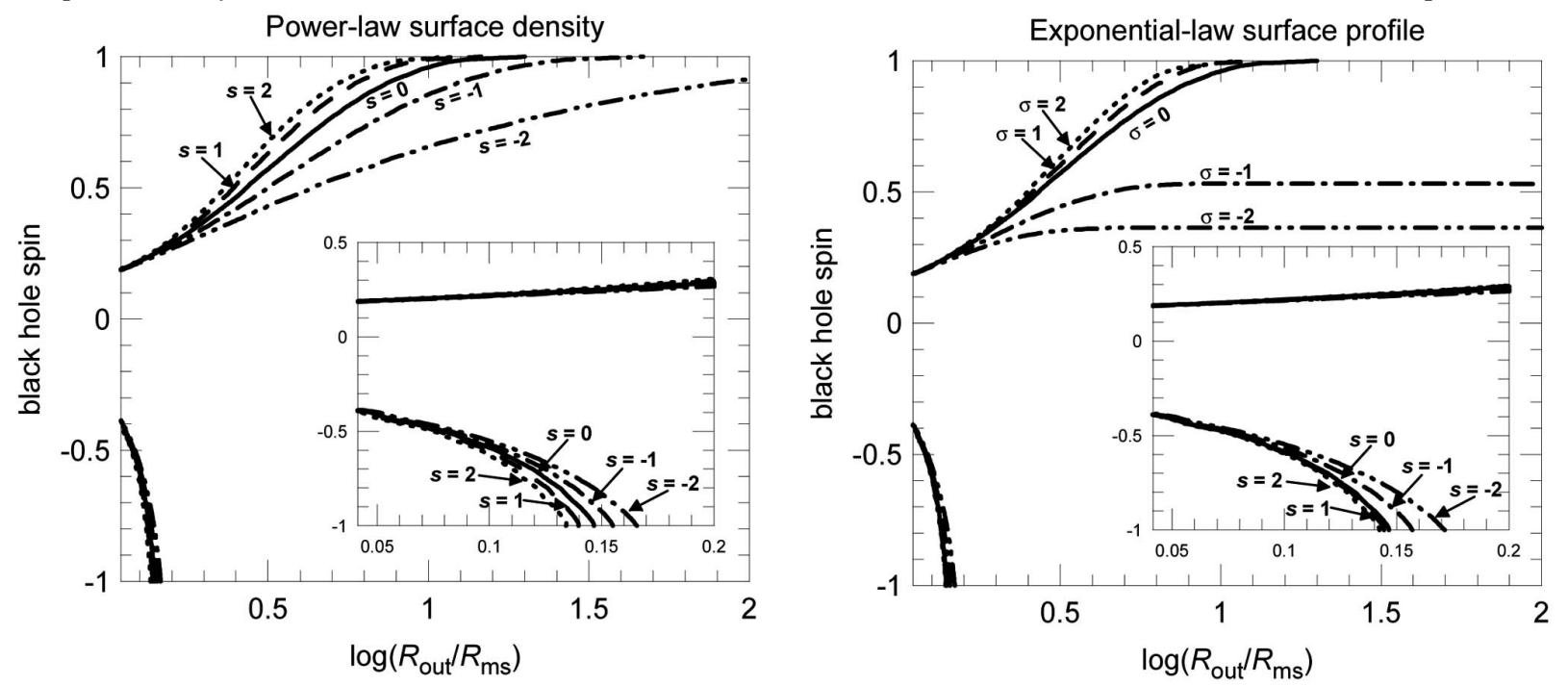

Fig. (1). Isocontours (in logarithmic scale) of the spin-induced precession period normalised by the BH mass (in units of yr $\mathrm{M}_{\odot}^{-1}$ ), for $3 \mathrm{C} 345$ disk models with power-law and exponential density profiles (left and right panels, respectively). In this case, solutions compatible with observations favor a system with $a_{*} \geq 0.20$ and $a_{*} \leq-0.35$, correspondingly. Indeed, in this source a full analysis shows that the spin of the black hole appears to be $a_{*}=-0.2$, that is $3 \mathrm{C}$ 345 harbours a counter-rotating (w.r.t. the accretion disk) Kerr black hole.

function like $\Sigma_{\mathrm{d}}(\xi)=\Sigma_{0} e^{\sigma \xi}$, where $\Sigma_{0}, \quad s$ and $\sigma$ are arbitrary constants. The results from the numerical calculation are shown in Fig. (1).

One can verify that in the case of 3C 345 the numerical solutions matching the observed $P_{\text {prec }} / M_{\mathrm{BH}}$ ratio favors the spin parameter in the range (prograde) $0.20 \geq a_{*} \leq-0.35$ (retrograde); for a power-law disk of index $s=-2$. These results combined with the independent search for the size of the B-P radius tightly constraint the $\mathrm{BH}$ spin in $3 \mathrm{C} 345$ to the value $a_{*} \simeq-0.20$, which means that it is counterotating w.r.t. disk angular momentum. It appears to be clear that the overall analysis based on B-P effect also provides an insight on the physical properties of its accretion disk.

Next we discuss the generation of GWs through the B-P mechanism. To this purpose, we first stress that the compactness of a rotating BH-Torus system (Eq. (1)), an example of a Kerr space-time, the ratio of rotational energy to linear size of the system is an invariant feature, say $C$, under rescaling of the $\mathrm{BH}$ mass. Formally, it reads

$$
\int_{0}^{E_{\mathrm{GW}}} f_{\mathrm{GW}} d E=C \simeq \sum_{i} f_{\mathrm{GW}_{i}} \Delta E_{i} .
$$

Because of this, one can expect that a physics, essentially similar to that developed by van Putten et al. [12] for the case of stellar-mass BHs as sources of Gamma-Ray Bursts and GWs, can take place in this size-enlarged version describing the SMBH-Torus in quasar 3C 345. Indeed, according to Ref. [13] a similar physics seems to dominate the source Sgr A*, which is said to harbour a SMBH-torus system in the center of our Galaxy. In the case of Sgr A*, the rise time for flaring should vary from half of, to at least a few times, the period of the vertical oscillations. This parameter has been estimated to be on the order of $\Delta P_{\text {vert }}^{F}=350-1000$ s. Meanwhile, the total duration of the flare $(F)$ should be about a few times the period of the radial oscillations, a parameter which has been estimated to be on the range $\Delta P_{\text {rad }}^{F}=40-120 \mathrm{~min}$ ! These timescales have been measured by both Chandra [14] and XMM-Newton Xray Telescopes $[15,16]$.

\section{GWS FROM ULTRA RELATIVISTIC ACCELERATION OF JET COMPONENTS IN AGN DYNAMICALLY DRIVEN BY B-P EFFECT}

The mechanism driving the ejection of jet components from AGN is not settled yet. Several possibilities have been suggested. Here we propose that the ejection process could benefit of the existence of a Lagrange internal point (in the static limit of the Kerr metric) created by the B-P effect at the transition radius. This is a placement wherein a force-free blob of accreted, piled-up material can be readily ejected. Among possible mechanisms driving the launching of superluminal components one can quote the instability of the vertical epicyclic oscillation of velocity $V_{31}$ at radius $r_{31}$, i.e. the $3: 1$ resonance! which can provide energy enough to seed an accretion-driven outflow from the AGN core. Nonetheless, several other alternative sources of energy to power the component ejection process are also gravitational. To quote one of those, one can consider BH-torus systems exhibiting the Aschenbach effect [16]. In such systems, one could benefit of the $\sim 2 \%$ reduction in the disk orbital velocity between radii $r_{\min }$ and $r_{\max }$ around the $\mathrm{KBH}$, which could amount as much as $4.3 \mathrm{MeV} /$ nucleon! However, here we do not discuss further long this issue since it is out of the scope of this paper.

Back to the mainstream of this Section, to describe the emission of GWs during the early acceleration phase of an ultra relativistic blob of matter ejected from the AGN core, i.e. piled-up mass that was initially trapped at the transition B-P region, one can start by linearizing Einstein's equations (hereafter we follow Ref. [17])

$G_{\mu v}=-k T_{\mu v}$, 
since we expect that the gravitational field produced by the ejection process at the B-P radius must be weak. To this purpose we use the expansion of the metric tensor:

$g_{\mu v} \simeq \eta_{\mu v}+h_{\mu v}$,

where $\eta_{\mu v}$ defines the Minkowsky metric, and $h_{\mu v}$ represents the space-time perturbation produced by the launching of the mass blob. In what follows we assume that the $g_{\mu v}$ metric has signature $(-+++)$, we use Cartesian coordinates $r^{\lambda}=t, r^{i}$ with $r^{i}=x^{1}=x, x^{2}=y, x^{3}=z$, and we also use geometric units in which $G=c=1$, so though $k=8 \pi$. We stress in passing that this GW signal is characterized by a gravitational wave memory, which means that the amplitude of the GW strain does not go back to zero as far as there is still available energy for the acceleration phase to go on. The GW signal will remain in that stage until gravitational energy is available in the radiating source (the launched blob). Considering the harmonic (Lorenz) gauge $h^{\alpha v}{ }_{v}=0$, one obtains

$\square h_{\mu v}=h_{\mu v, \beta}^{\beta}=16 \pi T_{\mu v}$,

where $h_{\mu v}=h_{\mu v}-\frac{1}{2} \eta_{\mu v} h_{\alpha}^{\alpha}$. Notice that one can figure out hereafter the knot as a "particle" of mass $M_{b}$ moving along the world line $r^{\lambda}(\tau)$ (with $\tau$ the proper time) and having an energy-stress tensor

$T_{\mu \nu}(x)=M_{b} \int V_{\mu} V_{v} \delta^{(4)}[x-r(\tau)] d \tau$

where $V^{\alpha}=d r^{\alpha} / d \tau$ is the particle 4-vector velocity.

Plugging this energy source into Eq. (5) leads to the retarded solution (a generalization of the Lienard-Wiechert solution)

$h_{\mu v}=\left.4 M_{b} \frac{V_{\mu}(\tau) V_{v}(\tau)}{-V_{\lambda} \cdot[x-r(\tau)]^{\lambda}}\right|_{\tau=\tau_{0}}$.

This result is to be evaluated at the retarded time, which corresponds to the intersection time of $r^{\alpha}(\tau)$ with the observer's past light-cone. Notice that the term in the denominator, $-V_{\lambda} \cdot[x-r(\tau)]^{\lambda}$, containing the 4-velocity, is responsible for the non vanishing amplitude ("memory") of the GW signal emitted during the launching of the blob from the AGN core.

This metric perturbation when transformed to the Lorenz gauge becomes $h_{\mu v}=h_{\mu v}-\frac{1}{2} \eta_{\mu v} h_{\alpha}^{\alpha} ;$ or equivalently

$h_{\mu v}=\frac{4 M_{b}}{-V_{\lambda} \cdot[x-r(\tau)]^{\lambda}}\left(V_{\mu}(\tau) V_{v}(\tau)+\frac{1}{2} \eta_{\mu v}\right)$.

Eq. (8) must be furtherly transformed into the transverse traceless (TT) gauge, i.e., $h_{\mu v} \rightarrow h_{\mu v}^{T T}$; which is the best suited to discuss the GW detector response to that signal. We invite the attentive reader to follow the detailed analysis of the detector response as given in Ref. [17] Such analysis shows that the maximum GW strain in the detector is obtained for a wave vector, $\vec{n}$, orthogonal to the detector's arm. In this case, the GW amplitude then reads

$h_{\max }=\frac{\gamma M_{b} \beta^{2}}{D}\left(\frac{\sin ^{2} \theta}{1-\beta \cos \theta}\right)$,

where $\beta=|\vec{v}| / c$, with $|\vec{v}|$ the particle's 3-D velocity, $\theta$ the angle between $\vec{v}$ and $\vec{n}, D$ the distance to the source and $\gamma$ the Lorentz expansion factor.

This result, obtained first in Ref. [17], shows that the GW space-time perturbation is not strongly beamed in the forward direction $\vec{n}$, as opposed to the electromagnetic radiation, for instance in gamma-ray bursts (GRBs). Instead, the metric perturbation at the ultra-relativistic limit (which is the case of superluminal components of AGN jets, but not for the run away galactic pulsars discussed in Ref. [18]) has a directional dependence which scales as $1+\cos \theta$. Indeed, because of the strong beaming effect, the electromagnetic radiation emitted from the source, over the same time interval, is visible only over the very small solid angle $\left(\theta \sim \gamma^{-1}\right)^{2}$, whereas the GW signal is observable over a wide solid angle; almost $\pi$ radians. Besides, the observed GW frequency is Doppler blueshifted in the forward direction, and therefore the energy flux carried by the GWs is beamed in the forward direction, too.

Table 1. Physical Properties of the C7 Component Ejected from Quasar 3C 345 for Julian Date 1992.05, as given in Ref. [19]

\begin{tabular}{|l|l|l|l|}
\hline \multicolumn{1}{|c|}{$\mathbf{N}\left(e^{-}\right)$} & \multicolumn{1}{|c|}{ Ang. Size } & \multicolumn{1}{c|}{ Diameter } & \multicolumn{1}{c|}{ Mass } \\
\hline \hline $\mathrm{cm}^{-3}$ & mas & $\mathrm{pc} \mathrm{h}^{-1}$ & $\mathrm{M}_{\odot} \mathrm{h}^{-3}$ \\
\hline $0.5-11$ & $0.20 \pm 0.04$ & 0.94 & $0.66 \rightarrow \simeq 1$ \\
\hline
\end{tabular}

The two asymptotic forms of the GW amplitude read (always for $\gamma \gg 1$ )

$$
\begin{aligned}
& h_{\max }(\theta \ll 1) \simeq \frac{4 \gamma M_{b} \beta^{2}}{D}\left(\frac{\theta^{2}}{\gamma^{-2}+\theta^{2}}\right) \\
& h_{\max }\left(\theta \gg \gamma^{-1}\right) \simeq \frac{2 \gamma M_{b} \beta^{2}}{D}(1+\cos \theta)
\end{aligned}
$$

Thus, if one applies this result to the information gathered from the quasar 3C 345 and for its C7 component (see Table I and Ref. [9]), then the mass of the ejected component is $M_{b} \simeq 1 \mathrm{M}_{\odot}$. Besides, if one assumes a distance $D=150 \mathrm{Mpc}$ [20] (instead of $2.5 \mathrm{Gpc}$ quoted by earlier references cited by Ref. [9]), the angle between the line-of-sight vs. jet component axis $\Theta_{1.0 . s}^{C 7 \leftrightarrow}=2^{\circ}$, the velocity ratio $\beta \sim 1$, and the Lorentz factor $\gamma=20$ [14], then one can see that the GW amplitude amounts

$h_{\mathrm{D}=150 M p c}^{3 C 345} \simeq 1.3 \times 10^{-20}$. 


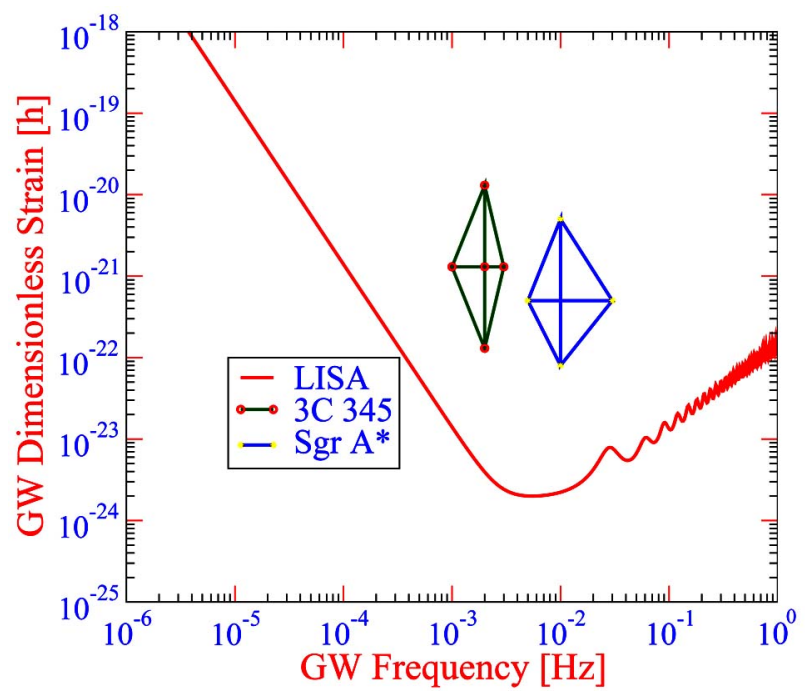

Fig. (2). Parameter space for the GW signals expected from acceleration of jet components in AGN 3C 345, and Sgr A*, the Galaxy source with a BH of mass $\mathrm{M}=3 \times 10^{6} \mathrm{M}_{\odot}$. The parameter space is fixed by the $\mathrm{X}$-ray observations quoted in the main text and the compactness of Kerr spacetime.

This is a GW amplitude which lies within the current strain sensitivity of LISA. ${ }^{2}$

Meanwhile, because of the invariant compactness of Kerr metric the azimuthal frequency scales inversely proportional to the $\mathrm{KBH}$ mass. That is,

$\frac{\mathrm{f}_{\mathrm{GW}} \mathrm{I}_{3 \mathrm{C} 345}}{\left.\mathrm{f}_{\mathrm{GW}}\right|_{\mathrm{SgrA}^{*}}}=\frac{M_{S g r A^{*}}^{B H}}{M_{3 C 345}^{B H}}$.

In other words, it is consistent with the Rayleigh criterion: in Kerr space-time, the energy per unit of angular momentum is small at large distances. Nonetheless, the characteristic acceleration time scale for the vertical launch of a given jet component during a flare is not properly known. A few AGN have such parameter reasonably estimated. For instance, Sgr A* accretion disk has a period of $<\Delta P_{\text {vert }}^{\text {osc }}>\simeq 500 \mathrm{~s}$ for the vertical oscillations, while the period for the radial oscillations is $\left\langle\Delta P_{\text {radial }}^{\text {osc }}\right\rangle \simeq 60 \mathrm{~min}$. Thus, the rise time for flaring must be either half of, or a few $(\leq 0.1)$ times the period of vertical oscillations $[14,15]$, while the total duration of a flare must be a few $(\leq 0.1)$ times the period of radial oscillations. In our studies we associate this time scale with the interval over which the peak acceleration of (and thus the maximum amplitude of the associated GW signal from) any ejected component is reached.

In this respect, since the B-P transition radius can be figured out as a Lagrange internal point from which each component is force-free ejected, then one can think of that the characteristic time for such acceleration must be a "universal constant", except for some extra factors on the order of 1 (see below the discussion on equipartition of

\footnotetext{
${ }^{2}$ Such GW signal would also be detected by Earth-based interferometers,
} but see next the discussion on the frequency of this signal. energy between a mass inhomogeneity in the disk and the torus mass). Indeed, regarding observations of most jets in the whole class of objects under analysis here, it appears that the distance from the host source that a given jet can reach does not depend on the mass of the system, but rather on the conditions in the circumjet medium, as density, for instance. That is, on ideal astrophysical conditions, all jets might reach the same distance from their host source. ${ }^{3}$

Thus, based on the Kerr compactness (Eq. (13)), and on the observational fact that the mass of the pc scale accretion torus in a number of AGN is, in average, les than a factor of 10 smaller than the mass of the SMBH in their host system, one can claim that the most likely astrophysical parameter playing some significant role in a pc scale torus make up by ${ }^{1} \mathrm{H}_{1}$ plasma is the sound speed (which involves several thermodynamical properties). It scales as the square root of the torus density, $\rho$, and also dictates the angular frequency of the warps in the disk [21], which hereby fixes the linear momentum to be delivered to a given jet component during its ejection. Such parameter depends on both disk height scale and radial size, which in the B-P effect scenario depends on both the spin parameter and the disk viscosity. (See the astrophysical foundations of this discussion in Ref. [9]).

Under this premise, one can take the Sgr A* flaring time scale as such "universal constant". In this way, one can estimate the GW frequency associated to the ejection of the C7 jet component of 3C 345

$$
f_{\mathrm{GW}}=\frac{1}{\left\langle\Delta P_{\mathrm{vert}}^{\mathrm{osc}}\right\rangle} \simeq \frac{1}{500 \mathrm{~s}}=2 \times 10^{-3} \mathrm{~Hz}\left(\frac{\rho_{\mathrm{S} g r A^{*}}^{d}}{\rho_{3 C 345}^{d}}\right)^{1 / 2}
$$

This frequency is clearly within the band width of LISA (see Fig. 2). Thus, by putting the information from both parameters into the strain spectral density diagram of the LISA GW observatory one can claim that the GW signals from acceleration of ultra relativistic jet components in this AGN 3C 345 can be detected.

Hence, by extending this result to each process of ejection of components from AGN; with the characteristic (typical) mass of any ejected component being about $M_{b} \simeq 1 \mathrm{M}_{\odot}$, one concludes that as far as the rise time (acceleration time scale) is lower than $10^{5} \mathrm{~s}$, which corresponds to the lowest frequency limit of LISA, such GW signals would be detectable for distances upto nearly the Hubble radius, i.e. $3 \mathrm{Gpc}$. Indeed, to perform a more consistent analysis, or a better characterization, of the GW signal produced during the early acceleration time of the ejected component, one can model the growth with time of the GW amplitude through a power-law of the sort $h(t)=h_{0}\left(t-t_{0}\right)^{n}$, where $t_{0}$ defines the time elapsed till the strain achieves its "final" steady-state, where no more GW radiation is emitted. In this way, one can evolve the $\mathrm{GW}$ waveform (Fourier transform of $h(t)$ ) so as to compare it

${ }^{3}$ One can invoke the Equivalence Principle to assert the existence of a universal time scale, despite that the $\mathrm{KBH}$ in $3 \mathrm{C} 345$ weighs $10^{3}\left(\mathrm{M}_{\odot}\right)$ times more than Sgr A*. 
with the strain-amplitude noise curve of LISA,. This analysis will be performed elsewhere (see the forthcoming paper in Ref. [9]).

3.1. A Potential Mechanism for Production of Jets in AGN: Surface Acoustic Wavelike (SAW) Behavior of Accretion Disk and Warps

In a very recent paper, a group of fluid dynamicists [22] exploit large accelerations associated with surface acoustic waves (SAWs) to drive an extraordinary fluid jetting phenomena. It is seen that laterally focusing of the acoustic energy to a small region beneath a drop placed on the surface causes a rapid interfacial destabilization. (One may envisage this phenomenon as a reminiscence of the dynamics of warps in an accretion disk. Indeed, in our view, this experimental result appears to be a Lab. scale-size version of the Sato mechanism relating the warp-warp interaction with the occurrence of QPOs in relativistic disks. The attentive reader is addressed to this visionary (yr 2004) paper [21]). It was found that above a critical Weber number $W^{4}$, an elongated jet forms for drops with dimensions greater than the fluid sound wavelength. Further increases in wave amplitude lead to single droplet pinch-off (a phenomenon which we claim is rather similar to jet component ejection) and subsequent axisymmetric breakup to form multiple droplets (a structure rather similar to the kiloparsec-scale jet of NGC 1068, see at $5 \mathrm{GHz}$, and $1.4 \mathrm{GHz}$, respectively. See Ref. [5]). All this phenomenology is described by a simple equation based on a momentum balance from which they predict the jet velocity. ${ }^{5}$

The vibrations, at a frequency of 30 megahertz, triggered different behaviors in the droplets, depending on the vertical acceleration of the waves, which the team controlled with slight changes in their amplitude. (In our view, in an association with the circumjet environment resistive effects, this may explain the quite reach diversity of jet morphologies seen in AGN surveys). For smaller amplitudes, the force was not strong enough to overcome the droplet's surface tension, so it merely oscillated in place, sloshing between a flattened shape and a "mountain" shape. (In our scenario, this peculiar behavior may be a down-size realization of the QPO and X-ray flaring stages of accretion disk in AGN, micro-qsrs, etc. Therefore, this phenomenology, properly up-scaled in size and mass, may be useful in the understanding of the dynamics of most accretion disks). For larger amplitude SAWs, the mountain

${ }^{4}$ The Weber number is a dimensionless number in fluid mechanics that is often useful in analysing fluid flows where there is an interface between two different fluids, especially for multiphase flows with strongly curved surfaces. It can be thought of as a measure of the relative importance of the fluid's inertia compared to its surface tension. The quantity is useful in analyzing thin film flows and the formation of droplets and bubbles. It is named after Moritz Weber (1871-1951) and may be written as:

$W e=\frac{\rho v^{2} l}{\sigma}$

where $\rho$ is the density of the fluid, $v$ is its velocity, $\iota$ is its characteristic length, typically the droplet diameter, and $\sigma$ is the surface tension.

${ }^{5}$ In the Lab. the researchers put two electrodes on a lithium-niobate piezoelectric surface, a material that converts electric voltage into SAWs. The electrodes, made from arrays of curved metal strips, or arcs, took up two 90degree sectors of a disk shape, with a hole in the center. The arrays focused SAWs from opposite directions into the center, where the researchers placed a 1- to 5-microliter drop of water, ethanol, methanol, or octanol. erupted into a jet 1 to 2 centimeters high, reaching speeds of several meters per second. The larger the SAW amplitude, the longer the jet. (Notice that a state of resonance in an accretion disk may mimic this feature. In such conditions it may impart to an ejected component a much larger velocity). At even higher amplitudes, the jet broke up into a series of smaller droplets. (In an accretion disk, a state of critical (Sato's warp-warp) resonance may produce similar effects, which then translates into even much larger ejection velocities). The largest SAWs caused the droplet to explode in a flurry of even tinier droplets, a process called atomization.

In all about, the reachness of this experimental result could be an insight into the overal dynamics of most astrophysical accretion disks and jets. In special, a significant step in this research topic could be to pursue studies considering droplets at smaller scales, where viscosity becomes more relevant. The outcome of which might lead to a significant understanding of the dynamics of warps in accretion disks dominated by B-P effect. Hence, and as a halfway conclusion, one can envision a fashion of putting into context the physics of droplets in the Lab. with the dynamics of accretion disks and the ejection of jet components in astrophysical systems as AGN, micro-qsrs and T-Tauri stars, among many other astrophysical systems.

\section{GWS FROM A MASS ORBITING THE SMBH AT THE TRANSITION RADIUS IN B-P DOMINATED AGN}

In the case of AGN driven by the B-P effect and suspended accretion any intrinsic mass-inhomogeneity $M_{b}$ in the torus, say a lumpiness or blob of mass in a warp trapped inside the B-P transition region, (which we assume to be eventually ejected as a jet component), generates a continuous GW signal. The typical strain can be estimated by using the Peters-Mathews weak field, quadrupole formula [23], which has been successfully applied to describe the dynamics of the PSR 1913+16 binary pulsar, and several other similar systems. Hence at the B-P radius a trapped mass produces a luminosity in GWs given as [24]

$$
L_{\mathrm{GW}}=2.5 \times 10^{57} \mathrm{erg}^{-1}\left[\frac{M_{\mathrm{B} H}}{10^{9} M_{\odot}}\right]^{5}\left(\frac{10 R_{g}}{R_{\mathrm{B}-P}}\right)^{5}\left[\frac{M_{b}}{1 M_{\odot}}\right]^{2}\left(\frac{10^{9} M_{\odot}}{M_{\mathrm{B} H}}\right)^{2}
$$

where $\left(\frac{M_{b}}{M_{\mathrm{BH}}}\right)$ is related to the chirp mass.

In this respect, the observations of the component $\mathrm{C} 7$, and the results of our B-P modeling of 3C 345 shown in Fig. (1), together with the observational parameters given in Table I, lead to infer that $M_{b} \sim 0.66 \mathrm{M}_{\odot}$. Hereafter, we approximate this mass inhomogeneity to $\simeq 1 \mathrm{M}_{\odot}$ in the computations below.

Thus, from this estimate and by recalling the relation between the GW amplitude $h$ and the GW luminosity $L_{\mathrm{GW}}$ one can compute the average (modulated) amplitude of each of the GW polarization modes $h^{+}$and $h^{\mathrm{x}}$, which then reads $[10,9]$. 


$$
<h_{\max }^{+}>\simeq 6 \times 10^{-22} \text { and }<h_{\min }^{+}>\simeq 2 \times 10^{-22}
$$

and

$$
<h_{\max }^{\mathrm{x}}>\simeq 4 \times 10^{-22} \text { and }<h_{\min }^{\mathrm{x}}>\simeq 2 \times 10^{-22},
$$

which were obtained for a distance $D=2.45 \mathrm{Gpc}$ to quasar 3C 345. Meanwhile, for the most quoted distance to 3C 345, $D=150 \mathrm{Mpc}$, the amplitudes given above should be multiplied by a factor of 16.7. Be aware of, that in order to search for this type of signals LISA should integrate it over several periods, which in the case of 3C 345 amounts to several years.

By analyzing the amplitudes of these strains one can conclude that such GW signals are broad-band and frequency-modulated [9]. This feature is due to the fact that both $h^{+}, h^{\mathrm{x}}$ have the same minimum value of its strain, while the corresponding maximum strain differs by a factor of 1.5 !

Meanwhile, the angular frequency of this GW signal can be computed as the Keplerian frequency at the BardeenPetterson radius, which in Ref. [3] we inferred to be $R_{\mathrm{B}-P}=14 R_{g}$, with $R_{g}=G M_{\mathrm{BH}} / c^{2}$. In this way, one gets

$f_{\mathrm{G} W} \simeq \frac{M^{1 / 2}}{R_{\mathrm{B}-\mathrm{P}}^{3 / 2}} \simeq 1.25 \times 10^{-7} \mathrm{H} z$

Therefore, with these characteristics in the case of $3 \mathrm{C}$ 345 such GW signals may not be detectable by LISA. Nonetheless, the result is quite stimulating since observations of AGN allow to infer that the masses for SMBHs range between $10^{6} \leq M_{\mathrm{B} H} \leq 10^{9} \mathrm{M}_{\odot}$, which means that for SMBHs with masses $M_{\mathrm{B} H} \leq 10^{7} \mathrm{M}_{\odot}$ the GW signal from a typical mass inhomogeneity $M_{b} \leq 1 \mathrm{M}_{\odot}$ orbiting around it, would be detectable by LISA. As an example, the dynamics around the $\mathrm{SMBH}$ in $\mathrm{Sgr} \mathrm{A}^{*}$ would clearly strike within the amplitude and frequency range of sensitivity (strain-amplitude noise curve) of the planned LISA space antenna. Certainly, a large sample of other AGN with similar characteristics as those of Sgr A* are relatively close-by to us ( $D \leq 100 \mathrm{Mpc}$ ). Thus, their continuous GW signals would be detectable with LISA.

\section{GWS FROM SUSPENDED ACCRETION STATE (OR TURBULENT FLOW) IN AGN WITH ACCRETION DISK PRECESSION DRIVEN BY B-P EFFECT}

A state of suspended accretion (SAS) develops when the inner face of the accretion torus emits Alfvén waves of negative angular momentum into the $\mathrm{BH}$ event horizon, while the outer face emits positive angular momentum Alfvén waves to infinity. The BH evolves by conserving energy and angular momentum consistent with the no-hair theorem. Hence, most of its spin energy is dissipated in the event horizon, having a large luminosity incident onto the torus inner face. The torus is hereby in a balance of energy and angular momentum flux received from the spin connection to the $\mathrm{BH}$. A fraction of the $\mathrm{BH}$ spin-energy is radiated mainly as GWs (plus neutrinos and particle winds), with a conversion efficiency factor defined as the ratio of the torus to the $\mathrm{BH}$ angular velocities. According to the Kerr solution of rotating black holes, the horizon surface encloses baryonic matter collapsed under its own gravitational forces, while leaving rotational energy accessible by the Rayleigh criterion. The latter is a consequence of the first law of thermodynamics [8].

Thus, the GWs luminosity, $L_{\mathrm{G} W}$, as a function of the $\mathrm{BH}$ luminosity, $L_{\mathrm{B} H}$, can be obtained from the equilibrium conditions for the torque and energy in the SAS (see details in Ref. [8]). As the equivalence in poloidal topology of pulsar magnetosphere indicates that most of the magnetic field on the SMBH horizon is anchored to the surrounding matter (the magnetosphere barrier quoted above, which the attentive reader can figure out through a perusal of the observations of the galaxy MCG-6-30-15), the total luminosity at horizon reads

$L_{\mathrm{BH}} \simeq L_{\mathrm{B}-\mathrm{P}}=\Omega_{\mathrm{B}-\mathrm{P}}\left(\Omega_{\mathrm{BH}}-\Omega_{\mathrm{B}-\mathrm{P}}\right) f_{\mathrm{B}-\mathrm{P}}^{2} A_{\mathrm{BH}}^{2}$,

where $\Omega_{\mathrm{B}-\mathrm{P}}$, corresponds to the angular frequency at the B-P radius, $\Omega_{\mathrm{BH}}$ defines the $\mathrm{BH}$ angular rotation, and $f_{\mathrm{B}-\mathrm{P}}$ the fraction of the $\mathrm{BH}$ total angular momentum flux incident on the torus that is carried on by $B_{\theta}$, which is the average $B$ field poloidal component, and $A_{\mathrm{BH}} \simeq b b\left\langle B_{\theta}\right\rangle$ the net magnetic flux supported by the torus. In this expression $b$ and $b$ are respectively the semi-major axes of the torus ${ }^{6}$.

Besides, notice that because of the different pointing of the gravitational force each face develops surface waves (in general, related to the disk warps and to the SAWs discussed above), retrograde for the inner and prograde for the outer face, as seen from the corotating frame. In other words, because the Reynolds number in the accretion disk goes much higher than $R e>2300^{7}$, the disk develops turbulence. Any coupling (often fully nonlinear) between these inner and outer waves (mediated by the $B$-field radial component $\left\langle B_{r}^{2}\right\rangle^{1 / 2}$ ) may lead to angular momentum transfer between both faces, which may result in a hydrodynamic instability known as Papaloizou-Pringle waves [26]. Such instability should appear whenever the torus slenderness ratio satisfies the condition

$\frac{b}{R_{\mathrm{B}-P}} \leq \frac{0.56}{m}$,

where $m=1,2,3 \ldots$ is the wave-mode. This allows the inner face to decrease its angular momentum and the outer face to increase its angular momentum. The extension of this theory to torii of finite slenderness, $b / R=(0-1)$, shows that the

${ }^{6}$ The attentive reader should recall that the torus is confined within the ISCO, with $R_{\mathrm{ISCO}} \simeq 6 \mathrm{M}$, and the B-P radius $R_{\mathrm{B}-\mathrm{P}} !$, and is elongated to and outwards from the $\mathrm{KBH}$, as discussed above.

${ }^{7}$ The Reynolds number $(R e)$ measures the relative weight of the viscous vs. inertial terms in the Navier-Stokes equation [25]. Laminar flow corresponds to a fluid flowing with Reynolds number $R e<2300$. Stokes equation is a particular case which describes fluids whose écoulement develops Reynolds number $R e<1$. 
$m \neq 0$ wave-modes become successfully unstable as the torus becomes more slender. We stress in passing that this coupling of modes could also be the origin of warps in the inner disk, which then would revolve with frequencies smaller $(<<0.1)$ than the torus rotation frequency around the Kerr black hole [21].

Now, to compute the GW emission from a turbulent accretion torus orbiting a $\mathrm{KBH}$, we follow hereafter the constructive review by van Putten [24]. That paper shows that gravitational waves are the dominant radiation channel for a strong coupling between the inner and outer face of the torus, as turbulence appears to be inevitable in flows around black holes. Such gravitational radiation appears to be given off mostly in quadrupole emissions. In fact, it comes out that [27] such emissions bring along multipole mass-moments (which sets it apart from Taylor-Couette flows in existing laboratory experiments, and seems to resemble the SAWs jetting mechanism quoted above), that are luminous in gravitational radiation with frequencies on the order of 1 $\mathrm{kHz}$ around stellar mass black holes and below $1 \mathrm{mHz}$ around $10^{6} \mathrm{M}_{\odot}$ supermassive black holes. This phenomenology might have already been seen in some of the recently discovered quasi-periodic oscillations (QPOs) around supermassive black holes [28, 29], like Sgr A*. As pointed out by van Putten [24], one of the most exciting prospects for astronomy in the present century is to directly "listen into" these turbulent flows in close proximity to an event horizon by upcoming gravitational wave observatories. Any such detection promises to identify physical properties that hitherto remain outside the realm of direct measurements, such as magnetic fields (presently resolved at about one-thousand Schwarzschild radii in selected AGN), the effective viscosity, structure and stability of the inner disk and the inner torus magnetosphere, magnetic disk winds and, ultimately, metrology on the central black hole itself.

Thus, for an equipartition between the quadrupole mass inhomogeneity $M_{b}$ and the torus mass $M_{T}$, that is, $M_{b} / M_{T} \simeq E_{B} / E_{k} \simeq 1 / 15$, the GW energy released comes out in the form of quasi-periodic, broad-line gravitational radiation with a luminosity

$$
\begin{aligned}
& L_{\mathrm{GW}}=\frac{32 c^{5}}{5 G}\left(\frac{M_{\mathrm{BH}}}{R_{\mathrm{B}-\mathrm{P}}}\right)^{5}\left(\frac{M_{b}}{M_{T}}\right)^{2} \simeq \\
& 3 \times 10^{39} \operatorname{erg~s}^{-1}\left(\frac{R_{\mathrm{B}-\mathrm{P}}}{10 \mathrm{R}_{g}}\right)^{3}\left(\frac{0.03 \mathrm{M}_{\mathrm{BH}}}{M_{T}}\right)^{3}\left(\frac{M_{\mathrm{BH}}}{10^{9} \mathrm{M}_{\odot}}\right)^{2}\left(\frac{10^{7} \mathrm{~K}}{T}\right)^{2}
\end{aligned}
$$

where $M_{\mathrm{BH}}$ refers to the mass of the $\mathrm{BH}$ in $3 \mathrm{C} 345, R_{\mathrm{B}-\mathrm{P}}$ denotes the disk B-P radius in the case of $3 \mathrm{C} 345, M_{T}$ denotes the mass of the inner disk or torus, and $T$ the accretion disk temperature.

The numerical figures for this GW luminosity deserves a brief discussion at this point. First, by recalling that a typical quasar radiates visible light with a luminosity of about $10^{47}$ erg $\mathrm{s}^{-1}$, and that such stage of continuous wind emission may last for about $1 / 3$ to several years ${ }^{8}$, one can infer that the tremendous turbulence in the inner torus dissipates into heat, shocks and magnetic field instabilities a huge amount of gravitational radiation energy. This is ease to check if one compares the above estimated GW luminosity to the $\mathrm{KBH}$ rotational energy extracted from slowing down the $\mathrm{KBH}$ spin, which is the radiation incident onto the inner face of the torus. Indeed, coming back to our model of quasar 3C 345 [9] and Fig. (1), the above analysis indicates that the spin energy available from a rotating $\mathrm{BH}$ in $3 \mathrm{C} 345$ of spin $a_{*}=0.2$ and mass $M_{\mathrm{BH}}=3 \times 10^{9} \mathrm{M}_{\odot}$ reads

$$
E_{\mathrm{KBH}}=0.29 a_{*} M_{\mathrm{BH}} c^{2} \simeq 3.6 \times 10^{62} \operatorname{erg}\left[\frac{a_{*}}{0.2}\right]\left(\frac{M_{\mathrm{BH}}}{10^{9} M_{\odot}}\right) .
$$

Setting $\eta=\frac{4}{9}\left[\left(\frac{R}{M}\right)^{\frac{3}{2}}+1\right]^{-2}=0.005-0.01 \quad$ [8] to denote the ratio of the angular velocity of the torus to that of the Kerr black hole, then the gravitational radiation energy released by the KBH spin in quasar 3C 345 can be estimated as $E_{\mathrm{GW}}=\eta E_{\mathrm{KBH}}$, i.e.

$$
E_{\mathrm{GW}} \simeq 5 \times 10^{60} \mathrm{erg}\left[\frac{\eta}{0.02}\right]\left(\frac{M_{\mathrm{B} H}}{10^{9} \mathrm{M}_{\odot}}\right),
$$

for the estimate of $\eta$ from our model of quasar 3C 345. Hence, by comparing to the GW luminosity in Eq. (22) one verifies that a huge amount of gravitational radiation energy is dissipated in any AGN turbulent torus.

Now, as in this $\mathrm{KBH}$-blob binary system the mass inhomogeneity $M_{b}$ is located at the B-P radius, the frequency of this $\mathrm{GW}$ radiation comes out to be

$$
f_{\mathrm{GW}}=1.7 \times 10^{-6} H z\left(\frac{10^{9} \mathrm{M}_{\odot}}{M_{\mathrm{BH}}}\right)^{-1}\left[\frac{6.2}{\left(\frac{R_{\mathrm{B}-\mathrm{P}}}{M_{\mathrm{BH}}}\right)^{3 / 2}+\left(\frac{a_{*}}{M_{\mathrm{BH}}}\right)}\right]
$$

For the parameters we obtained in modeling the quasar $3 \mathrm{C}$ 345 this corresponds to a GW signal out of the bandwidth of sensitivity of the planned LISA observatory. But once again notice that for an AGN with $\mathrm{BH}$ mass $M_{\mathrm{BH}} \leq 10^{7} \mathrm{M}_{\odot}$ the GW signal may be detected by LISA. ${ }^{9}$ It worths to recall that detection of the light curve in gravitational radiation is of interest, as it features a negative chirp associated with the expansion of the ISCO, during relaxation of the Kerr spacetime to a nearly Schwarzschild space-time. In virtue of this feature, the late-time frequency of gravitational wave emission provides a novel metrology of the mass of the black hole, according to [24]

$$
M_{\mathrm{BH}} \simeq 5.9 M_{\odot}\left(\frac{f_{\mathrm{GW}}}{1 \mathrm{kHz}}\right)^{-1} .
$$

\footnotetext{
${ }^{8}$ The attentive reader must bear in mind that the characteristic time scale of AGN activity is about $10^{5}$ years!

${ }^{9}$ For stellar mass black holes powering long GRBs, the GW emissions will be detectable by Advanced LIGO-Virgo within a distance of about 100 $\mathrm{Mpc}$, corresponding to one long GRB per year.
} 
Meanwhile, GW signals that extend over a finite bandwidth ( $B=0.1$ ) from a source at a distance $D$, with a ( $\Delta T_{\text {int }}$ ) time integration of $L_{\mathrm{GW}}$ over one year, give a characteristic strain amplitude

$$
\begin{aligned}
& h_{\text {char }}=\frac{\sqrt{2}}{\pi D} \sqrt{\frac{d E}{d f}}=3.1 \times 10^{-22} \\
& {\left[\frac{100 \mathrm{M} p c}{D}\right]\left[\frac{0.1}{B}\right]^{1 / 2}\left[\frac{R_{\mathrm{B}-P}}{10 \mathrm{R}_{g}}\right]^{3 / 2}\left[\frac{M_{\mathrm{B} H}}{10^{9} \mathrm{M}_{\odot}}\right]^{-1 / 2}\left[\frac{10^{7} \mathrm{~K}}{T}\right]\left[\frac{\Delta T_{\mathrm{int}}}{1 \mathrm{y} r}\right]^{1 / 2}}
\end{aligned}
$$

where we use the distance to quasar 3 C $345 D=150 \mathrm{Mpc}$. (For the formerly quoted distance to $3 \mathrm{C} 345, D=2.5 \mathrm{Gpc}$, one must divide this GW amplitude by a factor 16.7). Notice that we also ignored a redshift factor $1+z$ for the nearby source AGN of interest.

For Sgr $\mathrm{A}^{*}$, this $h_{\text {char }}$ might strike the threshold of sensitivity of LISA, depending on the approximations used. However, it most likely remains undetectable for extragalactic AGN harbouring SBMHs, as illustrated by the analysis above for quasar 3C 345. Aside from this, if this GW signal is detected, LISA will open a unique window to studying turbulence at high Reynolds number around SMBHs.

\section{CONCLUSIONS}

From the above analysis one can conclude that any AGN for which the disk and/or jet precession are driven by the Bardeen-Petterson effect becomes a natural environs for a suspended accretion state to develop. This B-P effect, in turn, drives each AGN to become a powerful source of bursts of gravitational waves from the ultrarelativistic acceleration of jet components, and of continuous broad-line gravitational radiation from both the orbiting of blobs of mass at the B-P radius; and the precession of the distorted, turbulent accretion disk. All such GW signals could be detected by GW observatories like LISA. One also notices that the prospective coeval detection of gravitational and electromagnetic radiations from these AGN may decisively help in picturing a consistent scenario for understanding these cosmic sources.

\section{REFERENCES}

[1] Caproni A. Observational evidence of spin-induced precession in active galactic nuclei. PhD Thesis, Universidade de São Paulo, 2004 (unpublished). http://arxiv.org/PS_cache/astro$\mathrm{ph} / \mathrm{pdf} / 0410 / 0410450 \mathrm{v} 1 . \mathrm{pdf}$

[2] Caproni A, Abraham Z. Observational evidence of spin-induced precession in active galactic nuclei. Astrophys J 2004a; 602: 625. (a) Caproni A, Abraham Z. Can long-term periodic variability and jet helicity in 3C 120 be explained by jet precession? Mon Not R Ast Soc 2004b; 349: 1218-26.

[3] Caproni A, Mosquera Cuesta HJ, Abraham Z. Observational evidence of spin-induced precession in active galactic nuclei. Astrophys J Lett 2004; 616: L99-L102.

[4] Caproni A, Abraham Z, Mosquera Cuesta HJ. The Influence of the black hole rotation on accretion disks and jets in active galaxies: the Case of NGC 1068. Braz J of Phys 2005; 35: 1167-71.

[5] Caproni A, Abraham Z, Mosquera Cuesta HJ. Bardeen-Petterson effect and the disk structure of the Seyfert galaxy NGC 1068. Astrophys J 2006a; 638: 120-24.

[6] Caproni A, Livio M, Abraham Z, et al. Warping and precession in galactic and extragalactic accretion disks. Astrophys J 2006b; 653: 112-6.
[7] Caproni A, Abraham Z, Livio M, et al. Is the Bardeen-Petterson effect responsible for the warping and precession in NGC 4258 ? Mon Not R Ast Soc 2007; 379: 135-42.

[8] van Putten MH. Proposed source of gravitational radiation from a torus around a black hole. Phys Rev Lett 2001; 87: 091101.

[9] Mosquera Cuesta HJ, Sanchez LA , Alfonso Pardo D, Caproni A, Abraham Z. Gravitational wave emission driven by BardeenPetterson effect in precessing (tilt) accretion disks and jets in active galactic nuclei and micro-quasars, 2010 in preparation.

[10] van Putten MH, Lee HK, Lee CH. Line-broadening in gravitational radiation from gamma-ray burst-supernovae. Phys Rev D 2004; 69: 104026.

[11] Bardeen JM, Petterson JA. The lense-thirring effect and accretion disks around kerr black holes. Astrophys J 1975; 195: L65.

[12] van Putten MH, Levinson A, Lee HK, et al. Gravitational radiation from gamma-ray bursts as observational opportunities for LIGO and VIRGO. Phys Rev D 2004b; 69: 044007.

[13] Liu S, Fromerth MJ, Melia F. Shadowing of the nascent jet in NGC 4261 by a line-emitting supersonic accretion disk. Astrophys J 2003; 596: 879.(a) Liu S, Melia F. Spin-induced disk precession in the supermassive black hole at the Galactic Center. Astrophys J 2002; 573: L23.

[14] Baganoff FK, Bautz MW, Brandt WN, et al. Rapid X-ray flaring from the direction of the supermassive black hole at the galactic centre. Nature 2001; 413: 45.

[15] Porquet D, Predehl P, Aschenbach B, et al. XMM-Newton observation of the brightest X-ray flare detected so far from Sgr a*. Astron Astrophys 2003; 407: L17.

[16] Aschenbach B. Measuring mass and angular momentum of black holes with high-frequency quasiperiodic oscillations. Astron \& Astrophys 2004; 425: 1075. (a) Stuchlik Z, Slany P, Torok G, Abramowicz MA. The Aschenbach effect: unexpected topology changes in motion of particles and fluids orbiting rapidly rotating Kerr black holes. Phys Rev D 2005; 71: 024037.

[17] Segalis EB, Ori A. Emission of gravitational radiation from ultrarelativistic sources. Phys Rev D 2001; 64: 064018.

[18] Mosquera Cuesta HJ, Bonilla Quintero CA. The gravitational wave signal of the short rise fling of galactic run away pulsars. JCAP 2008; 0811:006. e-Print: arXiv: 0711.3046 [astro-ph].

[19] Hirotani K, Iguchi S, Kimura M, Wajima K. Pair plasma dominance in the parsec-scale relativistic jet of 3C 345. Astrophys J 2000; 545: 100.

[20] Burbidge G. NGC 6212, 3C 345, and other quasi-stellar objects associated with them. Astrophys J 2003; 586: L119-L122.

[21] Kato S. Wave-warp resonant interactions in relativistic disks and kHz QPOs. Publ Astron Soc Japan 2004; 56: 559-567. (a) Ibrahim A, Sato K, Shibata K. Long term simulations of astrophysical jets. Prog Theor Phys Supp 2004; 155: 343.

[22] Tan MK, Friend JR, Yeo LY. interfacial jetting phenomena induced by focused surface vibrations. Phys Rev Lett 2009; 103: 024501.

[23] Peters PC, Mathews J. Gravitational radiation from point masses in a Keplerian orbit. Phys Rev 1963; 131: 435.

[24] van Putten MH. Nonthermal transient phenomena around rotating black holes. 2009. arXiv:0905.3367. (a) van Putten MH. Gammaray bursts: LIGO/VIRGO sources of gravitational radiation. Phys Rep 2001; 345: 1.

[25] Papaloizou JCB, Pringle J. The dynamical stability of differentially rotating discs with constant specific angular momentum Month Not R Ast Soc 1984; 208: 721. (a) Papaloizou J CB, Terquem C. On the dynamics of tilted discs around young stars. Mon Not R Ast Soc 1995; 274: 987.

[26] Batchelor GK, An Introduction to Fluid Dynamics, Cambridge: Cambridge University Press 1967. (a) Guyon E, Hulin J-P, Petit L. Hydrodynamique physique. CNRS Editions - EDP Sciences, 2e ed. 2001, 1re ed. 1991.(b) Happel J, Brenner H. Low Reynolds number hydrodynamics. New York: Prentice Hall 1965.

[27] Bromberg O, Levinson A, and van Putten MH. The gravitationalwave spectrum of a non-axisymmetric torus around a rapidly spinning black hole New Ast 2005; 11: 619. See also van Putten $\mathrm{MH}$ and Levinson A. Calorimetry of Gamma-Ray Bursts: Echoes in Gravitational Waves. Astrophys J 2001; 555: L41. 
[28] Gierli n' ski M, Middleton M, Ward M, and Done C. A periodicity of 1hour in X-ray emission from the active galaxy RE J1034+396. Nature 2008; 455: 369. e-Print 2008; arXiv: 0807.1899 [astro-ph].

[29] Gupta AC, Srivastava AK, Wiita PJ. Periodic oscillations in the intra-day optical light curves of the blazar S5 0716+714. Astrophys J 2009; 690: 216. (a) Rani B, Wiita PJ, Gupta AC. Nearly periodic fluctuations in the long-term x-ray light curves of the blazars AO 0235+164 and 1ES 2321+419. Astrophys J 2009; 696: 2170. (b) Gupta AC, Wiita PJ, Pandey JC, et al. A Quasi-Periodic Oscillation of 30 Minutes in the X-ray Light Curve of the BL Lac S5 0716+714. e-Print 2009; arXiv: 0906.3077 [astro-ph].

Received: May 20, 2011

(C) Mosquera Cuesta et al.; Licensee Bentham Open.

This is an open access article licensed under the terms of the Creative Commons Attribution Non-Commercial License (http://creativecommons.org/licenses/by-nc/3.0/) which permits unrestricted, non-commercial use, distribution and reproduction in any medium, provided the work is properly cited. 